\title{
Neue US-Richtlinien zur allergischen Rhinitis
}

\section{Die American Academy of Allergy, Asthma and Immunology hat zusammen mit der American College of Allergy, Asthma and Im- munology neue Richtlinien zur Diagnose und Behandlung der aller- gischen Rhinitis herausgegeben. Gewarnt wird darin vor der kritik- losen Verordnung von älteren Antihistaminika, die aus Kosten- gründen wieder verstärkt von den Behörden gefordert wird.}

Herausgekommen ist ein umfassendes über 50seitiges Werk, das alle Aspekte der Erkrankung beleuchtet, an der in den USA 20 bis 40 Millionen Menschen oder 10 bis $30 \%$ der Erwachsenen sowie $40 \%$ der Kinder leiden.

So streng wissenschaftlich sich die Richtlinien geben - alle Empfehlungen werden durch wissenschaftliche Studien untermauert - so sehr zielen die Aussagen doch auf ein politisches Umfeld. Denn die Behandlung der allerg i s $\mathrm{c} h$ e $\mathrm{n}$ Rhinitis ist auch in den USA zu einem Politikum geworden. Die Health Maintenance Organisations setzen die USÄrzte unter Druck, aus Kostengründen vermehrt wieder Antihistaminika der ersten Generation zu verordnen.

\section{Deutliche Kritik: Gespart wird an der falschen Stelle}

Diese Praxis wird jedoch von AAAAI und ACAAI offen abgelehnt. Die Richtlinien stellen klar: Die Antihistaminika der zweiten Generation sind Mittel der ersten Wahl bei der Behandlung der allergischen Rhinitis. Die Risiken der Mittel der ersten Generation seien nicht mehr hinnehmbar.

Verschiedene Studien hätten gezeigt, daß diese Mittel ein „kausaler Faktor für tödliche Verkehrsunfälle“" sind. Das Risiko von Unfällen am Arbeitsplatz sei sogar höher als nach der Einnahme von Narkotika und sedativen Hypnotika. Die Antihistaminika der ersten Generation würden bei Kindern zu Lernstörungen führen. Bei Akademikern könnten sie einen Karriereknick auslösen. Eine besondere Gefahr liege darin, daß viele Patienten die Einschränkungen der Leistungsfähigkeit nicht spürten und sich selbst deshalb überschätzten. Hinzu komme, daß die gleichzeitige Einnahme von anderen zentral aktiven Substanzen wie Alkohol, Schlafmitteln und Antidepressiva die negativen Auswirkungen auf die Leistungsfähigkeit ,potenziert".

\section{Auch die Kombination alte und neue H1-Blocker wird abgelehnt} Eine Absage wurde der Strategie erteilt, aus Kostengründen Antihistaminika der ersten und zweiten Generation miteinander $\mathrm{zu}$ kombinieren. Ein geläufiger Kompromiß ist etwa, dem Patienten für den Morgen ein nichtsedierendes Mittel der zweiten Generation zu verordnen und ihn abends ein Mittel der ersten Generation einnehmen zu lassen. Dies sei sinnlos, weil die Sedation bis in den nächsten Ta $\mathrm{g}$ nachwirke.

Die nasale Applikation von Antihistaminika wird als wirksame Behandlung der allergischen Rhinitis bezeichnet. Der zusätzliche Nutzen: Die Nasenschleimhäute schwellen ab. Die Behandlung sei aber nicht sehr pop u 1 ä r. Viele Patienten würden sich über den bitteren Geschmack beklagen. Wegen der Resorption der Mittel sei eine Sedation nicht ausgeschlossen, wenn Mittel der ersten Generation intranasal eingenommen würden.

Zurückhaltend ist die Bewertung der nasalen Steroide. Die lokalen Nebenwirkungen seien zwar gering, es könne aber $\mathrm{zu}$ einer Irritation der Schleimhaut bis hin $\mathrm{zu}$ Blutungen kommen. Die Patienten sollten darüber aufgeklärt werden und das Nasenseptum müßte regelmäßig untersucht werden. Nasale Steroide seien jedoch eine Möglichkeit, die Gabe von oralen Steroiden so lange wie möglich hinauszuzögern.

Die systemische Therapie wird als notwendig erachtet, wenn die Symptome sehr schwer und für den Patienten nicht mehr erträglich sind, sowie zur Behandlung einer sehr ausgeprägten Polyposis nasi. Von Steroidinjektionen - in den USA offenbar eine weit verbreitete Therapie - wurde in einer Pressemitteilung dringend abgeraten. Diese Behandlung sei nun wirklich nicht angemessen.

Positiv beurteilt wird die intranasale Applikation von Cromoglicinsäure. Die geringen Nebenwirkungen sprächen für dieses Mittel, es sollte möglichst schon vor Einsetzen der Symptome eingenommen werden. Wesentlicher Nachteil für viele Patienten sei die viermal tägliche Applikation. Auch intranasal applizierte Anticholinergika könnten das Ausmaß der Rhinorrhö mildern. Der Nachteil: Ein Austrocknen der Nasenschleimhaut sei nicht ausgeschlossen.

\section{Abschwellende Mittel nur mit Einschränkung empfohlen}

Die orale Gabe nasenschleimhautabschwellender Mittel wie Pseudoephedrin und Phenylpropanolamin wird zwar nicht generell abgelehnt. Aber die Nebenwirkungen wie Schlaflosigkeit oder Appetitverlust und Nervosität müßten bedacht werden. Bei bestimmten Begleiterkrankungen wie z.B. Herzrhythmusstörungen, Angina pectoris, aber auch bei Hochdruckerkrankungen oder Hyperthyreose sollten die Mittel nur mit Vorsicht eingesetzt werden.

(rme)

Quellen: Dykewicz MS, Fineman S: Executive Summary of Joint Task Force Practice Parameters on Diagnosis and Management of Rhinitis. Ann Allergy Asthma Immunol 1998; 81 Joint Task Force Algorithm and Annotations for Diagnosis and Joint Task Force Algorithm and Annotations for Diagnosis an Management of Rhinitis. Ann Allergy Asthma Immunol 1998; Task Force summary statements on Diagnosis and Management of Rhinitis. Ann Allergy Asthma Immunol 1998; 81 474-7. - Dykewicz MS, Fineman S, Skoner DP et al.: Diagnosis and management of rhinitis: complete guidelines of the Joint Task Force on Practice Parameters in Allerg y, Asthma and Immunology. American Academy of Allerg y, Asthma, and Immunology. Ann Allergy Asthma Immunol 1998; 81: 